\title{
THE THORACIC SURGERY FOUNDATION FOR RESEARCH AND EDUCATION
}

\section{April 2000 Alley-Sheridan Fellows}

I April 2000, the second level of executive course education, entitled "Building the New World of Health Care," was offered for the first time. This course will involve participants in preparing a plan for

\section{Herbert Dan Adams, MD}

Evansville, Indiana

Thomas Victor Bilfinger, MD

Stony Brook, New York

Robert S. Boova, MD

Bryn Mawr, Pennsylvania

Robert W. Emery, MD

Minneapolis, Minnesota

Richard Engelman, MD

Springfield, Massachusetts

Alex J. Furst, MD

Miami, Florida

Leigh I. G. Iverson, MD

Oakland, California

Raj B. Lal, MD

Lombard, Illinois

Edwin Tutt Long, MD

Kansas City, Missouri

John C. Myers, MD

Rockford, Illinois redesigning the health care system in the United States. The Foundation named the following individuals to receive Alley-Sheridan Scholarships to attend this course in April 2000.

Ranjit K. Rath, MD

Cincinnati, Ohio

Robert L. Replogle, MD

Chicago, Illinois

Jeffrey B. Rich, MD

Norfolk, Virginia

Richard G. Rouse, MD

San Antonio, Texas

Meredith L. Scott, MD

Orlando, Florida

Richard J. Shemin, MD

Boston, Massachusetts

Alan J. Spotnitz, MD

New Brunswick, New Jersey

Victor Frederick Trastek, MD

Scottsdale, Arizona

Harold C. Urschel, Jr, MD

Dallas, Texas 